Revue d'histoire de l'Amérique française

ZRS REVUE D.HISTOIRE DE L'AMÉRIQUE FRANÇAISE

\title{
Le Père Pierre-Joseph-Marie Chaumonot, missionnaire de la Huronie (suite et fin)
}

\section{André Surprenant}

Volume 7, numéro 4, mars 1954

URI : https://id.erudit.org/iderudit/301622ar

DOI : https://doi.org/10.7202/301622ar

Aller au sommaire du numéro

Éditeur(s)

Institut d'histoire de l'Amérique française

ISSN

0035-2357 (imprimé)

1492-1383 (numérique)

Découvrir la revue

Citer cet article

Surprenant, A. (1954). Le Père Pierre-Joseph-Marie Chaumonot, missionnaire de la Huronie (suite et fin). Revue d'histoire de l'Amérique française, 7(4),

505-523. https://doi.org/10.7202/301622ar d'utilisation que vous pouvez consulter en ligne.

https://apropos.erudit.org/fr/usagers/politique-dutilisation/ 


\title{
LE PERE PIERRE-JOSEPH-MARIE CHAUMONOT, MISSIONNAIRE DE LA HURONIE*
}

\author{
(suite et fin)
}

\section{CHAPITRE IV}

\section{DESTRUCTION DE LA HURONIE}

La guerre avec les Iroquois datait de la fondation même de Québec. En effet, dès 1609, Champlain et deux Français accompagnaient les Algonquins dans une attaque contre les Iroquois ${ }^{1}$. Champlain, alors, avait posé un geste très lourd de conséquences. Il est vrai que, tôt ou tard, il aurait fallu en venir à cette grave décision, car les Iroquois bloquaient le commerce des fourrures, commerce vital pour la petite colonie naissante.

En 1611, Champlain entreprenait une autre expédition. Cet état d'hostilité devait se prolonger presque indéfiniment et tenir continuellement en alerte la Nouvelle-France et les missions. C'est que Champlain s'était attaqué à la Nation la plus puissante, la plus fière, la plus féroce peut-être ${ }^{2}$.

Ces Iroquois occupaient la côte méridionale du lac Ontario depuis Niagara, et ils s'étendaient à l'est jusqu'à la rivière Hudson. D'après le Père Martin, ils n'avaient pas la passion de s'enrichir ou d'étendre leur domaine, mais celle de dominer ${ }^{3}$.

* Voir Revue d'Histoire de l'Amérique française, juin 1953: 64-87; septembre 1953: 241-258; décembre 1953: 392-412.

1. Léo-Paul Desrosiers, Iroquoisie (Montréal, 1947), 45-48.

2. Voir l'origine de cette nation d'après Diamond Jenness dans L.-P. Desrosiers, Iroquoisie, 17.

3. Félix Martin. éd. et trad., Relation abrégé de Quelques Missions des Péres de de la Compagnie de Jesus dans la Nouvelle-France par le Père Francois-Joseph Bressani de la même Compagnie (Montréal, 1877), 84. 
En 1642, Le Père Jogues tomba en leurs mains". Le Père Bressani subit le même sort en 1644 . Son martyre, extrêmement cruel, provoqua l'admiration du monde. Le Pape Innocent $\mathrm{X}$ baisa ses cicatrices avec respect et lui dit affectueusement: "Vous avez été mutilé pour la publication de l'Évangile; vous ne devez pas être privé de l'honneur d'offrir le Saint-Sacrifice"s. Bressani lui-même déclarait au Père Général: "Je n'aurais jamais cru que l'homme eût la vie si dure ${ }^{6}$."

Les périodes de trêve et de combat se succédaient ainsi, sans que le temps d'accalmie soit toutefois bien long. Par exemple, la paix conclue, lors de la venue de sept Iroquois, le 22 février $1646^{7}$, fut rapidement rompue lorsque les Iroquois martyrisèrent le Père Jogues, le 18 octobre 1646, et tuèrent son compagnon, Jean de la Lande ${ }^{8}$.

Dès lors, la guerre prit une tournure décisive; enivrés de leurs succès, les Iroquois allaient s'acharner, avec rage et cruauté, a détruire toute la Huronie. Déjà, nous l'avons dit, le village de SaintIgnace I s'était déplacé près de la maison Sainte-Marie; Brébeuf avait alors pris soin de ce bourg, tandis que Chaumonot s'occupait du bourg de la Conception, depuis avril 1648 . "Cette mission de la Conception, affirme Ragueneau, est la plus féconde de toutes, et pour le nombre des Chrestiens et pour leur zele: leur Foy y paroist auec auantage, leur sainteté est respectée mesme de Infideles, trois des principaux capitaines et plusieurs gens considérables y viuent dans un exemple qui presche plus que nos paroles: en vn mot la Foy de cette Eglise iette dans tout le reste du pays, vne bonne odeur du Christianisme"10. C'est de là que Chaumonot devait

4. Voir le récit de sa captivité et de sa délivrance dans Q, 2 (1643): 63-69; 74-82.

5. Rochemonteix, 2: 43.

6. Ibid., 41.

7. Q, 2 (1646): 6.

8. Voir Q, 2 (1647): 2-3; 36-42.

9. Jacques Douard avait été assassiné le 28 avril 1648, Q, 2 (1648): 77-78. Or, Chaumonot écrit: "Après la mort du petit Jacques Douard assassiné l'an passé ié me souviens d'avoir offert à Dieu en holocauste ce que j'avais de plus cher... entre autres... estoient les Chrestiens de la Conception dont j'avais le soin. Carayon, 137-138.

10. Q, 2 (1648): 60. 
voir crouler toutes les missions environnantes. Le 4 juillet 1648, le bourg de Saint-Joseph fut entièrement dévasté et son missionnaire, Antoine Daniel, indignement tué dans l'exercice même de ses fonctions sacerdotales ${ }^{11}$.

Antoine Daniel, qui avait été un des premiers compagnons de Chaumonot et son initiateur, lui apparut par deux fois. Ragueneau, sans porter de jugement, raconte ces apparitions:

Ce bon Pere s'apparut aprés sa mort à un des nostres par deux diuerses fois. En l'vne il se fit voir en estat de gloire, portant le visage d'vn homme d'enuiron trente ans, quoy qu'il soit mort à l'âge de quarante-huict. La plus forte pensée qu'eut celuy auquel il s'apparut, fut de lui demander comment la diuine Bonté auoit permis que le corps de son seruiteur fust traitté si indignement aprés sa mort, et tellement reduit en poudre, que mesme nous n'eussions pas eu le bonheur d'en pouuoir recueillir les cendres. Magnus Dominus et laudabilis nimis, respondit-il. Oüy, Dieu est grand et adorable à tout iamais: il a ietté les yeux sur les oppobres de ce sien seruiteur, et afin de les recompenser en Dieu, grand comme il est, il m'a donné quantité d'ames qui estoient dans le Purgatoire, lesquelles ont accompagné mon entrée et mon triomphe dans le Ciel.

Vne autre fois il fut veu assister à vne assemblée que nous tenions, touchant les moyens d'aduancer la Foy en ces Pays; et alors il paroissoit nous fortifiant de son courage, nous remplissant de ses lumières et de l'esprit de Dieu dont il estoit tout inuesty ${ }^{12}$.

Le Père qui continue l'autobiographie de Chaumonot, relate le fait de façon différente:

Chaumonot ajoutoit seulement qu'à la vue de ce bienheureux tant de choses lui vinrent à l'esprit pour les lui domander, qu'il ne savoit par où commencer son entretien avec ce cher défunt. Enfin, lui dit-il: "Apprenez moi, mon Père ce que je dois faire pour être agréable à Dieu". "Jamais répondit

11. Q, 2 (1649): 3-5. Près de 700 personnes tuées ou prisonnières.

12. Q, 2 (1649): 5. Voir aussi le récit, à peu près identique, de Marie de l'Incarnation dans ES, 4: 229-230. Le fait est raconté par le même Ragueneau dans une lettre au P. Carafa, de Sainte-Marie aux Hurons, le 1 er mars 1649, Auguste Carayon, éd., Première Mission des Jésuites au Canada, Lettres et documents inédits, 242-243. C'est aussi de cette époque que date l'apparition de saint Ignace à Chaumonot. Voir AB, 2: 13. 
le martyr, ne perdez le souvenir de vos péchés." C'est ce que le $\mathrm{P}$. Chaumonot a exécuté depuis très fidèlement, puisqu'il ne trouvoit point l'oceasion de se confesser pour la première fois à quelque Prêtre que ce fut qu'il ne lui déclarât tous les plus gros péchés de sa vie. Et même tous les jours, il ajoutoit à l'accusation de ses nouvelles fautes la confession de quelques uns des péchés qu'il auoit commis avant son entrée en religion.

Son confesseur ayant eu crainte une fois qu'il ne fit cela par scrupule, il lui répondit: "Mon Père, je ne suis point scrupuleux, grâces à Dieu! mais je ne me confesse jamais de ce qu'il y a de plus criminel en moi et de plus humiliant dans ma vie, que je ne ressente sur le champ un surcroît de grâces, de paix et de consolation dans mon âme: aussi ai-je encore autant de répugnance et de peine à faire ces sortes d'aveux de mes péchés et de mes misères, que la première fois que je m'en suis confesse." Voilà sans doute bien profiter des visites des gens de l'autre monde ${ }^{13}$.

Le 16 mars 1649, c'était au tour de la mission de Saint-Ignace II, depuis un an à peine, sous les soins de Chaumonot, de tomber aux mains des Iroquois $^{14}$. Le même jour, furent martyrisés les PP. Brébeuf et Gabriel Lalemant ${ }^{15}$.

La réaction des missionnaires, au plus fort de cette tempête, dépasse la petite sagesse humaine; il faut élever son jugement au niveau supérieur du sermon de la montagne: "Heureux serez-vous lorsqu'on vous outragera, vous persécutera"16. Voilà l'esprit authentique de cette déclaration de Ragueneau:

Quoy qu'il arriue, ce nous doit estre assez qu'il en tire sa gloire; et s'il luy plaist augmenter la foy de ces peuples, multipliant ses croix et sur eux et sur nous, nostre cœur y est préparé, nous les embrasserons avec ioye, et nous luy dirons sur la montagne du Calvaire d'aussi bon cœur que s'il nous auoit transportez sur la montagne de sa gloire, Bonum est nos hic esse. Ie parle de la sorte à cause que je crains qu'on ne craigne

13. AB, 2: 11-12. Pascal disait: "On expie nos péchés à force d'y penser" et "Tes péchés tu les comprendras à mesure que tu les expieras".

14. Q, 2 (1649): 10-13.

15. Q, 2 (1649): 13-25. A propos de Gabriel Lalemant, Chaumonot écrivit: "J'ay eu l'honneur d'estre enuiron trois semaines durant Maistre en la langue Huronne de son Nepveu (Jérôme Lalemant), Q, 2 (1649): 30 et R.G. Thwaites, éd., The Jesuit Relations and Allied Documents, 34: 219.

16. Luc, VII, 20-23. 
par trop pour nous, Aestimati sumus sicut oves occisionis, sed in his omnibus superamus, propter eum qui dilexit nos. Depuis la naissance de Christianisme, et depuis que Jésus-Christ n'a rachepté le monde que par son sang respandu sur la croix, nous sommes asseurez que la Foy n'a esté plantée en aucun lieu du monde qu'au milieu de croix et de souffrances, Ainsi ces désolations nous consolent, et au milieu de la persécution, dans le plus fort des maux qui nous attaquent et des plus grands malheurs dont on nous puisse menacer, nous sommes tous remplis de ioye et nostre cour nous dict que iamais Dieu n'a eû vn amour plus tendre pour nous, que celuy qu'il a maintenant $t^{17}$.

"Précieux holocauste de ces vertueux Pères (Brébeuf, Lalemant etc.), continue Chaumonot, que ne puis-je vous faire continuer en ma personne! ce sera quand il plaira à Dieu. Tous tant que nous sommes icy, nous n'avons iamais plus aimé nostre vocation qu'apres auoir veu qu'elle nous peut esleuer iusques à la gloire du martyre; il n'y a que mes imperfections qui m'en puissent faire quitter ma part..." ${ }^{18}$.

Dieu demandait le dépouillement total, la nuit des sens, dont parle saint Jean de la Croix. A cet appel, les missionnaires répondaient: "Ita, Pater". Telle fut aussi la réponse de Chaumonot:

...ie me souviens d'auoir offert à Dieu en holocauste ce que j'auois de plus cher en ce monde, dans la pensée qu'il me venoit qu'il n'y auoit rien, pour précieux qu'il fust, dont nous ne deussions aimer l'aneantissement, pourveu que d'iceluy quelque gloire en reuinst à Dieu; entre autres choses que i'offrois à Dieu comme celle que je cherissois le plus au monde, estoient les Chrestiens de la Conception dont j'auois le soin, et puis la maison de Saincte-Marie: le Bon Dieu a accepté mon offrande. Tous mes pauvres Chrestiens de la Conception à la réserue de 3 . ou 4 . ont été tuez, ou pris captifs par les Iroquois, et la maison de saincte Marie a esté détruite, quoy que plus doucement, qu'à ce que je m'estois resolu dès longtemps auparauant en mes méditations ${ }^{19}$.

Cette attitude, familière pour les Saints, se présente comme un

17. Q, 2 (1649): 25-26.

18. Q, 2 (1649): 28.

19. Q, 2 (1649): 28. 
acandale pour ceux du dehors: méprisés, battus, chassés et pourtant toujours joyeux, parce qu'ils ont été jugés dignes de subir des outrages pour le nom de Jésus. "Mais celui-là seul peut comprendre le bonheur de souffrir pour le Christ qui l'a véritablement aimén". Les PP. Brébeuf, Gabriel Lalemant, Daniel, Jogues, eux, avaient saisi le sens de cette réalité. Ils avaient vraiment compris "qu'il n'y a pas de plus grand amour que de donner sa vie pour ses amis" ${ }^{21}$ ".

La mission de la Conception étant ainsi isolée ${ }^{22}$, trois cents guerriers de ce bourg sortirent, le 17 mars, à la rencontre des Iroquois, pour les surprendre. Mais, ces derniers, plus rusés et en bien plus grand nombre que les Hurons, les tuèrent ou emmenèrent tous prisonniers ${ }^{23}$.

Ce fut vers la minuit (deux jours après la défaite), explique Chaumonot, qu'on nous l'annonça et tout aussitôt voilà dans toutes les cabanes, des pleurs, des sanglots, des cris lamentables. On n'entendoit partout que des femmes qui rogrettoient leurs maris, des mères qui pleuroient leurs fils, et d'autres parents qui s'attristoient de la mort ou de la captiuité de leurs proches. Là dessus un vieillard craignant avec sujet que les Iroquois ne vinssent enlever le bourg, qui étoit sans dófense par la perte de ses braves, commença à courir de tous côtés en criant à pleine tête: "Fuyons! fuyons! sauvonsnous? Voici l'armée ennemie qui vient nous prendre". A ce cri je sors dehors je parcoure les cabanes pour baptiser les catéchumènes, pour confesser les Néophytes et pour armer de la prière les uns et les autres. En faisant ainsi le tour de la bourgade, je m'aperçus que tout le monde l'abandonnoit pour se réfugier chez une nation qui étoit à onze grandes lieues de notre demeure. Je suivis ces pauvres fugitifs dans la vue de les aider pour leur salut et comme je ne pensai pas même à

20. J. Huby, trad. et com. Evangile de Saint-Marc (Collection Verbum Salutis II, Paris, 1927), 263. "Les croix du Christ sont fréquentes, mais elles sont toutes aimables, et quoi qu'il n'y ait ici aucune satisfaction pour les sens l'esprit néanmoins y vit plus content que s'il possédait tous les trésors de l'Europe", M. de l'Inc., ES. 3: 264 .

21. Jean, XV, 13.

22. "Ce lieu est situé au milieu du pays, sur la coste d'une belle rivière, qui n'ayant pas de longueur plus d'un quart de lieue, ioint ensemble deux lacs, l'un qui 'estend a l'Occident, tirant un peu vers le Septentrion, qui pourroit passer pour une mer douce l'autre qui est vers le Midi, dont le contour n'a guere moins de deux lieues, $Q, I$ (1640): 63 .

23. AB, 1: 62. 
prendre aucune provision je fis tout ce chemin sans boire et sans manger et même sans être las. Je ne songeai en marchant et je ne m'occupai qu'à consoler mon troupeau, à instruire les uns, à confesser les autres et à baptiser ceux qui ne l'étoient pas encore. Comme l'hiver duroit encore, je fus obligé de leur conférer le baptême avec de l'eau de neige que je faisois fondre entre mes mains. Ce qui fit mieux connoitre qu'en ce voyage les forces que j'avois me venoient d'en haut, c'est qu'un francois qui se trouva de la bande et qui étoit d'une complexion incomparablement plus forte pensa mourir en chemin, n'en pouvant plus de faiblesse, de lassitude et d'épuisement ${ }^{24}$.

Très impressionnant ce tableau du pasteur qui court après ses brebis, et qui s'oublie totalement pour ne songer qu'à leur bien-être.

Ces Hurons avaient été pris, en effet, d'une panique folle et leur triste exode ne faisait que commencer ${ }^{25}$. Parti de la Conception avec ses Hurons, le 19 mars, pour aller se réfugier chez la nation du Petun, Chaumonot émigra de nouveau, le 1er mai ${ }^{26}$, cette fois vers l'île Saint-Joseph (Ahoendoe), où déjà plusieurs sauvages s'étaient retirés.

"En moins de quinze jours, écrit Ragueneau, nostre Maison de Saincte Marie se veid dépoüillée de tous costez, et l'vnique qui resta sur pied dans ces lieux de terreur, plus exposez aux incursions de l'ennemy, ceux qui auoient quitté leurs anciennes demeures, y ayans mis le feu eux-mesmes, crainte qu'elles ne seruissent de retraite et de forteresse aux Iroquois:"27.

Aussi, douze capitaines, "les plus considérables", proposèrent au Père Ragueneau, un départ général pour l'île Saint-Joseph. Ils parlèrent pendant trois heures avec une "éloquence aussi puissante pour nous fléchir que l'art des Orateurs en pourroit fournir au milieu de la France ${ }^{28}$ ".

24. AB, 1: 62-64. Voir aussi Jones, 382.

25. "les vns s'estans iettez dans des montagnes que nous appellons la Nation du Petun...; les autres ayans pris party dans vne Isle que nous nommons l'Isle S. Ioseph, où nous commençasmes, il y a prés d'vn an, vne nouuelle Mission... les autres... dans des Isles plus éloignées de nostre grand Lac... nous tascherons d'establir nostre principale demeure... dans vne Isle que nous nommons l'Isle de Saincte Marie, Q, 2 (1649): 26.

26. Q, 2 (1649): 29. Voir aussi Carayon, 139.

27. Q, 2 (1649): 25.

28. Q, 2 (1649): 27-28. 
D'abord d'avis contraire, les Pères acceptèrent finalement la proposition des sauvages. Le 15 mai, ils incendièrent eux-mêmes leur résidence Sainte-Marie. Avec émotion, le Père Ragueneau raconte cette scène pénible:

Mais il fallut, à tous tant que nous estions, quitter cette ancienne demeure de saincte Marie; ces edifices, qui quoy que pauures, paroissoient des chefs-d'œuure de l'art aux yeux de nos pauures Sauuages; ces terres cultivées qui nous promettoient vne riche moisson. Il nous fallut abandonner ce lieu ${ }^{29}$, que ie puis appeller nostre seconde Patrie et nos delices innocentes, puis qu'il auoit esté le berceau de ce Christianisme, qu'il estoit le temple de Dieu et la maison des serviteurs de IesusChrist: et crainte que nos ennemis trop impies, ne profanassent ce lieu de saincteté et n'en prissent leur auantage, nous mismes le feu nous mesmes et nous vismes brusler à nos yeux, en moins d'vne heure, nos trauaux de neuf et dix ans ${ }^{30}$.

Enfin, entre cinq et six heures du soir, le 14 juin 1649, eut lieu le dernier départ. Missionnaires et derniers sauvages s'embarquèrent sur un petit vaisseau construit sur les lieux mêmes.

Ie me jettay, explique Ragueneau, auec la plus grande part des autres, sur des arbres de cinquante à soixante pieds de longueur, que nous auions abattus dans les bois et que nous traisnâmes dans l'eau, les lians tous ensemble pour nous faire un plancher flottant sur cet element infidelle, comme autrefois nous auions veu en France on conduisoit le bois flotté dessus les eaux. Nous voguasmes toute la nuit sur nostre grand Lac, à force de bras et de rames; et le temps nous estant fauorable, nous abordames heureusement au bout de quelques iours dans une isle où les Hurons nous attendoient, et qui estoit le lieu où nous avions pris le dessein de nous reunir tous ensemble, pour en faire une isle Chrestienne ${ }^{31}$.

Jamais, assure Ragueneau, "nous ne fusmes plus contens, et iamais, nous n'auons eu de subjects d'une plus sensible tristesse ${ }^{32}$ ". Affirmation confirmée par Chaumonot, dans une lettre au Père Lalemant: "Tous tant que nous sommes de Pères, icy, nous n'auons iamais plus

29. "non sans quelque petit retour de la Nature", Q, 2 (1649): 30.

30. Q, 2 (1650): 2-3.

31. Q, 2 (1650): 3.

32. Q, 2 (1649): 30-31. 
aimé nostre vocation qu'après auoir vue qu'elle nous peut eslever iusques à la gloire du martyre; il n'y a que mes imperfections qui n'en puissent faire quitter ma part. Hélas, mon Reuerend Pere, que i'ay besoin d'humilité et de pureté de cœur pour pouvoir aspirer à l'honneur que le bon Dieu a fait à vostre nepveu! si V.R. la demande pour moy au bon Iesus par les merites de ses quatre grand seruiteurs les PP. Iogues, Daniel, de Brebeuf et Lalemant, i'espers qu'elle me l'obtiendra, et en suite le bon Iesus me pourroit bien faire la grace de mourir pour l'aduancement de son Royaume ${ }^{33}$."

Les missionnaires se remirent énergiquement à la tâche. Une nouvelle résidence, Sainte-Marie II, fut commencée sans délai et terminée en novembre. Elle était située sur la côte est de l'île, face à une grande baie et non loin du rivage ${ }^{34}$. De plus, les Pères aidèrent à fortifier le bourg des Hurons, contigu à leur demeure; tls dressèrent eux-mêmes des bastions pour defendre les approches de l'habitation. Tous les Français y allèrent de leurs forces, de leurs armes, de leur courage même; ils étaient prêts à exposer leur vie pour la défense de ce bourg "vrayement Chrestien"35".

Le sol, demeuré longtemps inculte, très peu travaillé par une population épuisée et plus occupée à s'installer, ne produisit pas suffisamment pour nourrir tout ce peuple affam $\hat{s}^{36}$; les aumônes des missionnaires demeuraient tout à fait insuffisantes. L'hiver devait être affreux:

C'est icy, rapporte Chaumonot, où ie vois vne partie des miseres que la guerre et la famine ont causées à ce pauure peuple désolé: leur nourriture ordinaire n'est plus que de gland ou d'vne certaine racine amere qu'ils nomment otsa, et bienheureux encore qui en peut auoir; ceux qui n'en ont pas viuent partie d'ail cuit sous les cendres ou dans l'eau sans autre sauce, et partie de poisson boucané, dont ils assaissonnent l'eau tout pure qu'ils boiuent, comme ils faisoient auparauant leur sagamité; il s'en trouue encore de plus pauures que tout cela, qui n'ont ny bled, ny gland, ny ail, ny poisson, et sont de pauures malades qui ne sauraient chereher leur vie.

33. Q, 2 (1649): 28-29. Martin, 100. Caravon. 138-130.

34. Jones, 383.

35. Q, 2 (1650): 3.

36. RA, 225. 
Adioustez à cette pauureté, qu'il faut qu'ils trauaillent à defricher de nouuelles forests, à faire des cabanes, et à faire des palissades pour se garantir, l'année qui vient, de la famine et de la guerre, en sorte que les voyans vous iugeriez que ce sont de pauures morts déterrez ${ }^{37}$.

Le Père Bressani, en résumant la Relation de 1650, ne parle pas autrement; il est même beaucoup plus réaliste:

C'était un spectable horrible de voir au lieu d'hommes, des squelettes moribonds, semblables aux ombres de la mort plutôt qu'à des corps vivants, aller et venir, et prendre pour se nourrir les choses les plus répugnantes à la nature. Ils arrachaient de la terre les cadavres que nous avions ensevelis de nos propres mains, car souvent les parents des défunts n'en avaient pas la force, et ils s'en nourrissaient ainsi que des restes de renards et des chiens. De quoi n'est pas capable un homme affamé? On sait que les Hurons, quand ils étaient infideles, mangaient les cadavres de leurs ennemis, mais ils éprouvaient autant d'horreur que les Européens à se nourrir de leurs compatriotes. Dans cette circonstance, la nécessité renversait toutes les lois. Des frères ont dévoré le cadavre de leurs frères, des mères ceux de leurs enfants, des enfants celui de leur père.

Une scène qui se renouvelait souvent, c'était de voir de petits enfants s'attacher encore au sein de leur mère après sa mort, des mères expirer avant d'avoir pu être délivrées, ou des enfants mourir sur leur sein, qu'elles offraient à ceux qui étaient plus âgés, mais dont ils tiraient plutôt du sang que du lait. Elles montraient une résignation si héroïque et si chrétienne, ce spectacle faisait couler des larmes d'attendrissement plus encore que de pitie ${ }^{38}$.

"Ie voudrois, reprend Chaumonot, pouuoir representer à toutes les personnes affectionnées à nos Hurons, l'état pitoyable auquel ils sont réduits: certainement elles ne pourroient se contenir de sangloter et de pleurer à chaudes larmes. Hélas! que ie leur dirois volontiers de la part de tout ce pauure peuple, Miseremini mei, miseremini mei, saltem vos amici mei, quia manus Domini tetigit me. Le tres-benin Iesus fut touché de compassion à la veuë d'vne seule veuue, dont on portoit le fils en terre; comment seroit-il possible

37. Q, 2 (1649): 29. Martin, 100-101. Carayon, 139.

38. RA, 226-227. Voir aussi $Q, 2$ (1650): 3-6. 
que ces imitateurs de Iesus-Christ ne fussent émeus à pitié à la veuë des centaines et centaines de veuues dont non seulement les enfans, mais aussi les parens ont esté outrageusement ou tuez, ou emmenez captifs, et puis inhumainement bruslez, cuits, déchirez et deuorés des ennemis ${ }^{39}$."

Les malheurs se suivaient, s'appelaient les uns les autres. La guerre avait provoqué l'exil, l'exil la famine, la famine les maladies contagieuses. De plus, les sauvages vivaient continuellement sous une tension nerveuse intense, s'attendant, nuit et jour, à être envahis $^{40}$. En dépit de ces menaces terribles, les pauvres Hurons, loin de maudire Dieu, le bénissaient à l'exemple du saint homme Job, et le remerciaient même. Jamais, en effet, ils n'avaient été si zélés pour entendre la messe, s'approcher des sacrements et écouter les prédications. L'horaire de la journée des missionnaires, transcrit par Ragueneau, le prouve abondamment. Après deux ou trois heures de prière et de méditation, prises sur les heures de sommeil, les Pères disaient la messe pour les sauvages. Pendant cet office, un prêtre dirigeait leurs prières. La messe était suivie d'une courte instruction pratique "pour qu'ils puissent saintement passer la journée". La chapelle était trop petite, les prédicateurs devaient recommencer ce petit sermon dix ou douze fois dans une même matinée, pendant que d'autres Pères entendaient les confessions et causaient avec les sauvages. De neuf heures du matin, jusqu'a deux heures avant la nuit, les Pères visitaient les cabanes. Ces visites, qui prenaient le reste de la journée, étaient suivies de la prière du soir, répétée comme le matin, de dix à douze fois. "Tousiours, déclare Ragueneau, la nuit nous surprenoit plutost que nous ne désirions; mais neantmoins nous la recevions auec amour, elle seule nous donnant le loisir de retourner auec Dieu; si toutefois on peut sortir de luy, lors qu'on ne parle que de luy, qu'on n'agit que pour luy, qu'on vit en luy, dans l'attente de ne mourir iamais pour autre que pour luy ${ }^{41} . "$

39. Q, 2 (1649): 29. Martin, 101. Carayon, 139-140.

40. Q, 2 (1650), 2-6.

41. Cette parole du Père Ragueneau se trouve dans $Q, 2$ (1650): 7. Et ce qui précède cette déclaration est un résumé de $Q, 2(1650): 5-7$. "Jamais nous n'avons recueilli de si grands fruits de nos travaux; jamais la Foi n'a poussé de si profondes 
Les quelques nouvelles venant de l'extérieur n'étaient pas de nature à réjouir et à calmer les angoisses des habitants de l'île SaintJoseph. En effet, le 7 décembre 1649, le Père Charles Garnier, missionnaire du bourg Saint-Jean, fut massacré avec la majorité des sauvages de l'endroit ${ }^{42}$. Le 8 décembre, le Père Chabanel était tué par un huron apostat ${ }^{43}$.

Le retour du printemps avait amené quelque espoir pour les sauvages. Les glaces étant fondues, ils sortirent de l'île pour trouver un peu de nourriture, glands, poissons, etc. ${ }^{44}$ Mais, comme l'explique très bien le Père Bressani, là où ils espéraient trouver la vie, ils ne rencontrèrent que l'esclavage ou la mort. Ils voulaient éviter de mourir de faim, et ils périrent dans les flammes. Ils tombèrent entre les mains de leurs ennemis, occupés à les rechercher de tous côtés, surtout pendant la nuit, et ils ne purent leur opposer aucune résistance, car ils étaient divisés en plusieurs bandes, et avaient avec eux leurs femmes et leurs jeunes enfants, qui ne servaient qu'à augmenter l'effroi et la confusion ${ }^{45}$.

A l'annonce de la marche de deux armées ennemies, les sauvages s'affolèrent. Deux capitaines, les plus anciens, rencontrèrent le supérieur, le Père Ragueneau, et lui tinrent ce discours émouvant. Nous le reproduisons en entier:

Frère, tes yeux te trompent lorsque tu nous regardes. Tu crois voir des hommes vivants et tu ne vois que des spectres et des âmes trépassés. Cette terre que tu foules au pieds, va

racines dans les cœurs; jamais le nom chrétien n'a été plus glorieux qu'au milieu des ruines de cette malheureuse nation. L'année dernière nous avons baptisé plus de trois mille sauvages. Nous touchons du doigt la vérité de cette parole de l'apôtre: Flagellat Deus omnem filium quem recipit", Ragueneau au Général, le Père Carafa, le 13 mars 1650, Rochemonteix, 2: 96.

42. Né à Paris, le 25 mai 1605 , entré chez les Jésuites le 5 septembre 1624 , il arriva \& Québec le 11 juin 1636 . Il fut martyrisé chez les Petuns par les Iroquois le 7 décembre 1649, Melançon, 34. Voir le récit de son martyre dans $Q, 2$ (1650): 8-16.

43. Noël Chabanel est né à Salses, diocèse de Mende, le 2 février 1613. Il entra dans la Compagnie de Jésus, à Toulouse, le 9 février 1630. Arrivé au pays le 15 aoat 1643 , il fut tué en haine de la foi par un huron apostat, près de l'embouchure de la rivière Nottawasaga, Ontario. Melançon, 19. Le récit de sa mort se trouve dans $Q, 2$ (1650): 16-19.

44. $Q, 2$ (1650): 23.

45. RA, 227-228. 
s'entr'ouvrir pour nous engloutir avec toi, afin que nous soyons au lieu qui nous est dû, parmi les morts. Il faut que tu saches, frère, que cette nuit, dans un conseil, on a pris la résolution d'abandonner cette île. La plupart de nous ont dessein de se retirer dans les bois, afin de vivre solitaires, et qu'homme du monde ne sachant où ils sont, l'ennemi ne puisse en avoir connaissance... D'autres disent tout haut, qu'ils vont mener leurs femmes et leurs enfants, pour se jeter entre les bras de l'ennemi, où ils ont un grand nombre de leurs parents qui les réclament, et qui leur donnent avis qu'ils aient à se sauver au plus tôt d'un pays désolé, s'ils ne veulent périr sous des ruines. Frère, que feras-tu, solitaire dans cette île, lorsque tout le monde t'aura quitté? Es-tu venu ici pour cultiver la Terre? Veux-tu enseigner à des arbres? Ces lacs et ces rivières ont-ils des oreilles pour écouter tes instructions? Pourrais-tu suivre tout ce monde qui va se disperser? La plupart trouveront la mort, où ils espèrent trouver la vie. Quand tu aurais cent corps pour te diviser en cent lieux, tu ne pourrais pas y suffire; tu leur serais à charge, et tu leur ferais horreur. La famine les suivra partout, et la guerre les trouvera.

Frère, prends courage; toi seul peux nous donner la vie, si tu veux faire un coup hardi. Choisis un lieu où tu puisses nous rassembler, et empêche cette dispersion. Jette les yeux du côté de Québec, pour y transporter les restes de ce pays désolé. N'attends pas que la famine et la guerre nous aient massacrés jusqu'au dernier.

$\mathrm{Tu}$ nous portes dans tes mains et dans ton cœur. La mort t'en a ravi plus de dix mille; si tu diffères davantage, il n'en restera plus un seul, et alors tu auras le regret de n'avoir pas sauvé ceux que tu aurais pu retirer du danger, et qui t'en présentent les moyens. Si tu écoutes nos désirs, nous ferons une église à l'abri du fort de Québec. Notre foi n'y sera pas éteinte. Les exemples des Français et des Algonquins nous maintiendront dans notre devoir. leur charité soulagera une partie de nos misères, et au moins y trouverons-nous quelquefois un morceau de pain pour nos petits enfants qui, depuis si longtemps, n'ont que des glands et des racines amères pour soutenir leur vie. Après tout, dussions-nous mourir là avec eux, la mort nous y sera plus douce qu'au milieu des forêts, où personne ne nous aiderait à bien mourir, et où nous craignons que notre foi ne s'affaiblisse avec le temps, quelque résolution que nous ayons prise de la chérir plus que nos vies ${ }^{46}$.

46. Nous avons suivi le texte de RA, 228-229. Il est également reproduit dans Q, 2 (1650): 24-25. 
Les Pères réfléchirent longtemps, revenant quinze et vingt fois sur le sujet; ils implorèrent le secours du ciel; ils firent les prières des quarante-heures et, finalement, ils décidèrent d'écouter la voix de Dieu, qui avait dû parler par la bouche de ces deux capitaines ${ }^{47}$.

Treize Pères, quatre frères, quarante-trois Français et environ trois cents sauvages, seuls survivants d'une nation autrefois très nombreuse, s'embarquèrent, le 10 juin $1650^{48}$, dans des canots, en route pour Québec ${ }^{49}$. "Ce ne fut pas sans larmes, commente Ragueneau, que nous quittasmes ce païs, qui possedoit nos cœurs, qui arrestoit nos espérances, et qui estant désia rougy du sang glorieux de nos freres, nous promettoit vn semblable bon-heur, nous ouuroit le chemin du Ciel et la porte du Paradis. Mais quoy! il faut s'oublier de soy-mesme, et quitter Dieu, pour Dieu, ie veux dire qu'il mérite luy seul d'estre seruy, sans la veuë de nos interests, fussent-ils les plus Saints que nous puissions auoir au monde ${ }^{50}$."

Odyssée de neuf cents milles, remplis de difficultés et d'embûches. Le convoi circula au milieu des ennemis, par la rivière des Français, le lac Nipissing, la rivière des Ottawas et le Saint-Laurent. Plusieurs firent naufrage tombant "dans des précipices aff reux, et dans le milieu des abismes". Au milieu du voyage, les exilés eurent une heureuse alarme. Deux groupes allaient être en présence et se préparaien't au combat, quand, de part et d'autre, on se reconnut. C'étaient le Père Bressani, quarante Français, vingt Hurons, qui, ignorant tous les malheurs précédents, allaient renforcer la colonie huronne. La petite troupe fit volte-face et revint sur ses pas. Tout le groupe passa deux jours à Montréal, où il fut reçu "auec vn cœur de Charité vrayement Chrestienne". Mais les sauvages ne voulurent pas demeurer à cet endroit, par crainte des Iroquois. Enfin, après une navigation d'environ cinquànte jours, Chaumonot et le groupe atteignaient Québec, le 28 juillet $^{51}$.

47. Voir RA, 229 et $Q, 2$ (1650): 25-26.

48. $Q, 2$ (1650): 1.

49. Jones, 401. Quarante-trois Français, soit vingt-deux donnés, onze domestiques, quatre enfants, six soldats, Jones, 401.

50. Q, 2 (1650): 26.

51. Pour ce voyage voir: $Q, 2$ (1650): 26-28; Rochemonteix, 2: 112-114; Jones, 401-402; Martin, 108; C.H. Laverdière et H.R. Casgrain, éd., Le Journal des Jésuites, 139, 142. 
La petite colonie vivotait. Elle devait maintenant accueillir près de quatre cents personnes, sans vivres, sans vêtements, sans abri. La réception n'en fut pas moins des plus cordiales. Les Hospitalières, les Ursulines, les Français les plus à l'aise, les Jésuites donnèrent sans compter.

Par les chemins, explique Ragueneau, nous les auons nourris; dans leur propre pais, Dieu nous fournissoit les moyens de soulager vne partie de leurs miseres; nous auons respandu pour eux nostre sang et nos vies: pourrions-nous apres cela leur refuser ce qui est hors de nous, qui puisse estre en notre pouuoir? Ils viennent tous les iours quérir chez nous la portion qu'on leur distribuë; ils se sont bastys eux-mesmes leurs cabanes, ils tascheront par leur trauail de chercher quelque partie de leur nourriture. Si apres nous estre espuisez, nous nous voyons dans l'impuissance de continuer nos charitez, et qu'ils meurent icy de famine proche de nos François, au moins aurons-nous cette consolation, qu'ils y mourront Chrestiens ${ }^{52}$.

Il faut dire que le spectacle devait être réellement affreux, pour que Marie de l'Incarnation écrive au sujet des Pères: "Les Révérends Pères qui sont descendus des Hurons et qui ont porté le poids de la tyrannie de ces barbares et qui se sont retirés ici des missions éloignées ont si épouvantablement souffert, qu'il n'y a pas de langue humaine qui le puisse exprimer", et encore: "Les Révérends Pères qui étaient demeurés vifs avaient plus souffert que ceux qui étaient morts. L'on voyait que c'étaient des personnes consommées et dans lesquelles Jésus-Christ vivait plus qu'elles ne vivaient en elles-mêmes. Leur sainteté était si visible à tout le monde que chacun en était ravi ${ }^{53}$." Témoignage splendide d'une mystique, seul témoignage qui ait, ici, une valeur.

C'était la fin d'une des missions les plus hérő̈ques qui furent jamais. Avec ces paroles toutes surnaturelles du Père Bressani, nous concluons:

Si le lecteur me demande quel sera le sort de cette Mission, si elle se rétablira jamais, s'il y a espoir que les Hurons et leurs missionnaires puissent y retourner un jour, je donnerai pour réponse ces paroles du Psalmiste: Les jugements de Dieu sont

52. Q, 2 (1650): 28.

53. ES, $4: 286,294 ; 2: 429$. 
un abîme insondable ${ }^{54}$. Cependant si l'on parvient à abattre l'orgueil des Iroquois, pourquoi cette belle Mission ne se rétablirait-elle pas? Je sais qu'il y a à cela de grandes difficultés; mais ce qui est impossible aux hommes est possible à Dieu, qui peut tout ${ }^{55}$. Et d'ailleurs, le monde ne sera détruit qu'après que l'Evangile aura été prêché en tous lieux. Or dans les contrées de l'Ouest, depuis le pays des Hurons jusqu'à la mer de Chine, on trouve des nations innombrables, auxquelles le Royaume de Dieu n'a pas encore été annoncé. Il faut donc qu'un jour l'Evangile arrive jusqu'à eux, lors même que ces Missions seraient interrompues quelque temps.

Dieu sait quand ce moment viendra. Il ne nous appartient pas de connâ̂tre le temps et les moments que le Père a fixé dans sa puissance ${ }^{56}$ mais il est juste que nous lui demandions avec instance, que son règne arrive au plus tôt, et que tous les peuples et que toutes les nations le bénissent, afin qu'il n'y ait qu'un seul troupeau et qu'un seul Pasteur et que tous ensemble nous chantions les louanges de Celui qui vit et règne dans les siècles des siècles ${ }^{57}$.

Restaient maintenant, en Nouvelle-France, trente missionnaires éparpillés dans diverses missions ou établis au collège de Québec, ou dans les résidences de Trois-Rivières, de Montréal, de Sillery ${ }^{58}$.

Chez les Hurons, Chaumonot avait passé onze ans de sa vie. Il lui avait fallu quitter ce pays bien-aimé. Il ne fut pas séparé, cependant, de ses chers Hurons, car le Père Lalemant le nomma spécialement pour s'occuper d'eux à Québec. Ainsi, il allait continuer son apostolat auprès de ceux, pour qui, à chaque instant, il immolait sa vie.

\section{CONCLUSION}

Aux yeux des Juifs, la mort du Christ sur la croix marque un échec total.

Saint François-Xavier, expirant presque seul dans la petite île de Sancian, face à la Chine, où il n'avait pu pénétrer, semble, aux yeux des sages de ce monde, avoir manqué sa vie.

54. $\mathrm{Ps}, \mathrm{XXXV}, 7$.

55. Luc, VXIII, 27.

56. Actes, 1, 7.

57. RA, 231-232.

58. RA, 232. 
Après un séjour de onze ans en Huronie, le retour du Père Chaumonot à Québec, revêt aussi les apparences d'un échec retentissant.

Comment alors juger l'apport de Chaumonot à la mission huronne? Des missionnaires qu'elle envoyait en Nouvelle-France, la Compagnie de Jésus exigeait une aptitude réelle pour les langues et surtout une vie intérieure intense. Ces deux exigences, Chaumonot les remplissait pleinement. Il a été avec Brébeuf "le meilleur linguiste de la Huronie" ${ }_{1}$; et il a possédé parfaitement l'esprit surnaturel requis.

Il est vrai, comme l'a écrit le Père de Rochemonteix, "qu'il était simple jusqu'à la crédulité, timide jusqu'à la peur ${ }^{2}$, d'une intelligence peu cultivée, d'une nature impressionnable, et d'un caractère où ne se distinguait rien de saillant ${ }^{3}$ ". Il est vrai que Chaumonot ne manifesta jamais une science extraordinaire; jamais, il n'a été supérieur; toujours il a agi en second; on ne lui connaît pas de méthode d'apostolat particulièrement originale, mais du moins avait-il ce zèle des âmes, cette "charité sans laquelle nous sommes des airains sonnants, des cymbales retentissantes, cette charité, sans laquelle nous ne sommes rien; cette charité qui est patiente, qui croit tout, espère tout, supporte tout, qui ne finira jamais"".

Et en cela, il a suivi l'exemple du Christ qui a montré l'impuissance radicale des grandeurs charnelles et des grandeurs de l'esprit (sans les condamner toutefois) à produire à elles seules des fruits surnaturels. Le Christ a manifesté la Charité et la Charité seule. Le Christ n'a jamais fait étalage de science, et pourtant, il savait tout.

1. Latourelle, 2: 250. Nous rappelons de nouveau ce paragraphe déjà cité: "Tous ceux même des nôtres qui apprendront jamais le Huron l'apprendront à la faveur des préceptes, des racines, des discours et de plusieurs beaux ouvrages qu'il nous a laissés en cette langue. Les sauvages eux-mêmes avouoient qu'il le parloit mieux qu'eux, qui se piquoient la plupart de bien parler, et qui parlent en effet avec beaucoup de pureté, d'éloquence et de facilité", AB, 2: 15.

2. "J'avois tant de répugnance à faire ces visites qu'à chaque fois que j'entrois dans les cabanes, il me sembloit que j'allois au supplice: tant j'appréhendois les railleries qu'il m'y fallait souffrir", $A B, 1: 53$; ...'mais je n'ai jamais été saisi d'aucune crainte dans toutes ces rencontres: en quoi j'ai reconnu une grâce de Dieu toute particulière puisque je suis timide de mon naturel", $\mathrm{AB}, 1: 68$.

3. Rochemonteix, 1: 399.

4. 1 Cor., XIII, 1-7. 
Dans la vie du Christ, seule apparaît la Charité, au point que l'on peut résumer sa vie entière par ce mot: "Il a aimé". La vie missionnaire de Chaumonot n'a été aussi qu'un acte d'amour continuel. Après les huit martyrs canadiens, dont l'Église a reconnu la grandeur, Chaumonot est peut-être le type le plus représentatif du "parfait missionnaire", titre que lui décerne d'ailleurs son supérieur, le Père Claude Dablon".

C'est ainsi que l'échec apparent du début, placé dans l'axe de la croix, se change en une réussite complète. Chaumonot, fidèle disciple du Christ, n'a pas été traité différemment du Maitre. Il a porté, lui aussi, sa croix. Car seule la passion du Christ, continuée dans son corps mystique qu'est l'Eglise, a converti et convertira le monde. Dieu l'a voulu ainsi. Le monde a été sauvé par la croix; à travers les siècles, l'Église recevra les fruits de salut et de rédemption par la même passion. C'est la loi: "Si le grain de blé tombé en terre, ne meurt pas, il reste seul; mais s'il meurt, il produit beaucoup de fruit ${ }^{6} . "$

La conversion des âmes, œuvre essentiellement surnaturelle, est donc seule l'œuvre de la charité. Cette charité, nous l'avons vu, Chaumonot la possédait éminemment. Cette partie de sa vie, que nous venons de raconter dans les chapitres précédents, et que nous résumons brièvement ici, en est le vivant témoignage.

Pierre Chaumonot vole quelques sous chez son oncle, curé de Châtillon-sur-Seine, où il est né, pour aller étudier au collège de Beaune; il s'enfuit avec le dessein très ferme de ne jamais revenir en arrière. Il continuera plutôt son chemin, préférant mendier misérablement. A Terni, où il trouvera finalement une situation au collège des Jésuites, nous voyons le gueux d'hier partager son argent avec les pauvres.

Touché par le sermon d'un prédicateur, il fait le vœu de quitter le monde pour devenir bientôt novice de la Compagnie de Jésus. A partir de ce moment, sa vie sera une montée continuelle, une purification constante, grâce à sa fidélité à la conduite de l'Esprit-Saint. L'humilité profonde qu'il développera, dès son noviciat, lui vaudra des faveurs insignes.
5. Martin, 244.
6. Jean, XII, 24. 
La Relation de 1636 de Brébeuf exerce sur lui une influence déterminante. Elle est à l'origine de sa vocation missionnaire. Il brale les étapes de la longue formation de Jésuite, pour devenir prêtre et se rendre en Nouvelle-France, dès 1639. Deux jours après son arrivée, il part pour le pays des Hurons, où il montre toutes les vertus des saints: une douceur inaltérable, une charité sans bornes, un zèle infatigable, une union continuelle avec Dieu, une confiance inébranlable, une foi vive, une humilité vraiment sincère. "Il a été faible avec les faibles, afin de gagner les faibles. Il s'est fait tout à tous, afin d'en sauver de toute manière quelques uns ${ }^{7}$."

La paix sereine qu'il éprouve, au milieu des difficultés inoules, loin de tous les charmes extérieurs de la vie, loin de toutes les occasions de vaine gloire, il la trouve dans cette mort au monde et à lui-même.

Les dangers l'animent, les famines, les guerres le rassurent, les souffrances le comblent, le martyre fait son attente. Attitude incompréhensible pour ceux-là seuls qui mettent tout leur espoir en cette vie mortelle, qui ne comprennent pas que le Père Chaumonot ait pu rechercher le martyre une infinité de fois et qu'il ait pu accepter avec joie les affronts, les persécutions, les calomnies, les plaies.

La Providence, qui avait appelé ses premiers compagnons, Brébeuf et Daniel, à la gloire du martyre, se contenta de ses ardents désirs et de son immolation volontaire et quotidienne à Jésus-Christ. Elle le réservait en effet pour une noble tâche, celle de diriger "le reste" des Hurons échappés aux Iroquois. Le martyre lui a manqué, mais lui n'a pas manqué au martyre.

Chaumonot n'était pas de ces âmes molles, dont parle Dante, que le ciel repousse et que l'enfer dédaigne. C'était une âme trempée, qui ne refusa jamais rien aux avances du Ciel, une âme qui possédait la générosité, l'ampleur, la fécondité. Chaumonot n'était pas un triste; il n'était pas un pessimiste. Il était solide, équilibré.

C"était un de ces "doux qui possèdent la terre", un de ces "purs qui voient Dieu".

André Surprenant, s.j.

7. 1 Cor., IX, 22-23.

8. Matt., $V, 4,8$. Nous terminons ici la première partie de la vie admirable du Père Pierre-Joseph-Marie Chaumonot. Nous avons déja résumé la deuxième partie de sa vie dans l'introduction. Voir Revue d'Histoire de l'Amerique francaise, (juin 1953), 65. 